$\begin{array}{ll}\text { Italique } & \text { Italique } \\ \text { Poésie italienne de la Renaissance }\end{array}$

V| 2002

Varia

\title{
Les poètes français et les anthologies lyriques italiennes
}

Jean Balsamo

\section{OpenEdition}

Journals

Édition électronique

URL : http://journals.openedition.org/italique/143

DOI : 10.4000/italique.143

ISSN : 1663-4438

Éditeur

Librairie Droz

Édition imprimée

Date de publication : 31 décembre 2002

Pagination : 9-32

ISBN : 2-600-00826-8

ISSN : $1423-3983$

\section{Référence électronique}

Jean Balsamo, «Les poètes français et les anthologies lyriques italiennes », Italique [En ligne], V | 2002, mis en ligne le 06 octobre 2009, consulté le 19 avril 2019. URL : http://journals.openedition.org/ italique/143 ; DOI : 10.4000/italique.143

(c) Tous droits réservés 


\author{
JEAN BALSAMO \\ LES POÈTES FRA N ÇA IS \\ ET LES ANTHOLOGIES LYRIQUES \\ I T A L I E N N E S
}




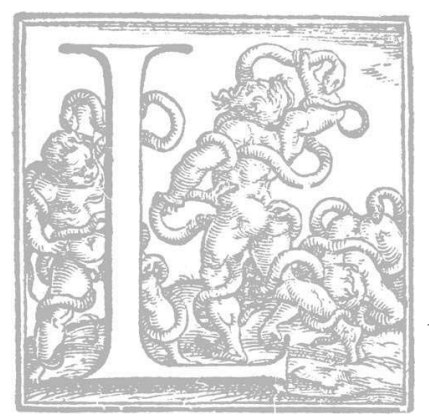

'Édition moderne de la première grande anthologie néo-pétrarquiste, les fameuses Rime diverse di molti eccellentissimi autori, due aux soins attentifs de Franco Tomasi et Paolo Zaja, ne saurait assez être louée pour la qualité de sa présentation, et parce qu'elle donne enfin à lire un recueil, rare, souvent allégué et finalement mal connu. ${ }^{\mathrm{T}}$

Ce sera l'occasion de proposer quelques remarques sur la diffusion de ce recueil, et plus généralement des anthologies poétiques italiennes au XVI $T^{e}$ siècle et leur réception en France. En 1909, dans une étude alors novatrice, Joseph Vianey mit en lumière certaines des sources littéraires où les poètes de la Pléiade avaient puisé: ${ }^{2}$ les anthologies de poésie lyrique, vénitiennes principalement, qui se multiplièrent à partir de I545, offrant des sonnets choisis de plusieurs centaines d'auteurs. L'ouvrage de Vianey, complété par une liste des principaux recueils, avait été permis par les travaux d'Hugues Vaganay, bibliographe et fin bibliophile qui avait su réunir une importante collection de ces livres rares. Grâce à lui, Vianey inventa la notion de pétrarquisme français, qu'il définissait comme l'influence des néopétrarquistes italiens, utilisant une notion tout autre que critique, qui interdisait de concevoir en termes dynamiques le travail de lecture et d'imitation auquel se livrèrent les poètes français. À la suite d'une tradition déjà longue, il faisait de cette influence l'expression d'un art de cour et la chargeait d'un certain nombre d'implications morales négatives. ${ }^{3}$ L'ouvrage de Vianey marqua plus d'un demi-siècle d'bistoire littéraire, et lui imposa ses conclusions: les poètes français n'auraient cessé de suivre les Italiens; ils avaient commencé par les néo-pétrarquistes de la fin du siècle précédent; Tebaldeo, Chariteo, Serafino dell'Aquila, en qui Vianey voyait le "pìre de presque tous les sonnettistes français»; au cours d'une deuxième période, ils auraient découvert l'école bembiste; après Is57 enfin, le goût maniéré des quatrocentisti et des poètes méridionaux serait revenu à la mode, et le grand modèle aurait été Angelo di Costanzo. ${ }^{4}$

Pour documentée qu'elle était, la thèse de Vianey ne pouvait pourtant qu'aboutir à une double aporie. Elle insistait sur le rôle de ces «modernes Italiens» que Du Bellay invoquait dans la Deffence comme les champions du sonnet,', et elle négligeait Pétrarque. À la suite de ces prémisses, Vianey négligeait nécessairement la rivalité qui opposait Ronsard et ses suiveurs au fondateur de la poésie toscane et le rôle que jouait la persona poétique de Pétrarque dans l'éthos d'une poésie lyrique fondée sur une référence explicite. Marqué par le mythe romantique de l'originalité, Vianey d'autre part ne pouvait pas comprendre l'esthétique de l'imitation à l'oeuvre dans la poésie du 
$X V T^{e}$ siècle, et il était conduit à interpréter en termes contradictoires de plagiat l'imitation des textes italiens, d'inexactitude ou d'infidélité le travail bien différent de variation élaboré à partir de ces modèles, dont les poètes français auraient suivi "linfluence" toujours délétère en termes de goût. L'écart nécessaire entre le modèle et le texte français ne pouvait lui apparaître que comme un défaut et nullement comme un élément dynamique dans la composition d'un poème dont l'enjeu était précisément de se démarquer de sa source. De surcroît, si Vianey procédait à certains rapprochements judicieux et documentés, nombre de ses analyses reposaient sur de simples analogies thématiques. Toutes les études de source, à sa suite, témoignent ainsi du même préjugé et des mêmes faiblesses. Outre des rapprochements forcés et des approximations, mis en évidence par une plus stricte confrontation des textes, ces études de sources ignorent à la fois la dimension topique de l'écriture poétique de la Renaissance et les phénomènes d'intertextualité. ${ }^{6}$

Plus que les poètes de la Pléiade eux-mêmes, que Vianey cherchait malgré tout de préserver du péché d'un "pétrarquisme" excessif, ce fut Desportes qui assuma pour l'ensemble de la critique tout le passif du legs néo-pétrarquiste, esthétique et moral. Jacques Lavand estimait au tiers des 63 I poèmes recueillis dans la dernière édition du poète la part des pièces "influencées" par les Italiens on imitées d'eux.7 Il négligeait de s'interroger sur l'utilisation que le poète avait faite de ces textes. La recherche des "sources" italiennes de Desportes dans les anthologies lyriques devint un exercice qui occupa les savants pendant longtemps. ${ }^{8}$ Il fallut attendre les travaux de Pizzorusso et de Graham, qui surent dépasser l'approche purement analogique et statistique des sources, pour voir rappeler les conditions d'une plus juste méthode d'interprétation et de comparaison, capable de conduire à des conclusions moins simplistes. ${ }^{9}$ Le "maniérisme" de Desportes et son prétendu mawvais-goût ne provenaient pas de l'imitation de Serafino, de Sasso ou de Tansillo; ils ressortissaient à l'écriture même du poète et aux enjeux propres à la poésie française, et partant, étaient à définir en d'autres termes. ${ }^{10}$

\section{La diffusion française des anthologies}

Hugues Vaganay indiquait dans son édition des Amours de Diane de Desportes que les recueils italiens étaient de son temps «à peu près introuvables en France»; ${ }^{\mathrm{II}}$ cette remarque avait déjà faite par Chamard. ${ }^{12}$ L'observation, bien qu'exagérée, est toujours d'actualité. Pour ne prendre en considération que les Rime diverse de 1545 et leurs rééditions de I546 et I549, on connaît à peine une douzaine d'exemplaires en France: Besançon, Nantes, Mazarine pour l'édition originale, Grenoble, La Rochelle, Paris BnF (2 exemplaires), Paris Arsenal, Montpellier (2 exemplaires), Toulouse pour les rééditions. 
Cette rareté actuelle avait une cause plus ancienne. Les anthologies poétiques italiennes semblent n'avoir circulé en France que de façon confidentielle. Toutes les bibliothèques françaises du XVI siècle sont loin d'avoir été recensées, et de surcroit, les inventaires qui en restent sont sommaires, les notaires et les libraires qui prisaient les livres mentionnaient le plus souvent en lots les volumes de petit format. Schutz ne recense qu'un exemplaire des Rime di diversi dans son enquête. ${ }^{3}$ On ne dispose d'autre part d'aucune indication sur la présence de ces livres dans les stocks des libraires parisiens, comme on ignore tout des circuits de leur distribution. ${ }^{14}$

L'exemplaire de la Mazarine porte la signature de l'bistorien Du Bouchet et la date de I633; il passa ensuite au couvent de Saint-Victor rien n'indique en revanche son premier possesseur. Celui de Toulouse, relié en vélin, porte sur le titre la date I592, et une mention en français, mais il reste anonyme, comme celui de La Rochelle. L'actuelle présence de la plupart des exemplaires dans les bibliothèques publiques tient pour une bonne part du collectionnisme des $X V I I I^{e}$ et XIX ${ }^{e}$ siècles, et ces exemplaires ont souvent pu être acquis tardivement en Italie. Le splendide exemplaire conservé à Grenoble, dans une reliure italienne de l'époque en veau blanc, au chiffre DD, provient du président D'Esery, qui l'a acheté pour 3 florins. Les recueils conservés à l'Arsenal ont été réunis par Nyon, le bibliothécaire du duc de La Vallière; la collection des Rime diverse (I546-I550) ainsi, en dépit d'une reliure uniforme en veau blond, est constituée de trois volumes de provenances diverses, qui semblent avoir été tardivement réunis: si le premier est entièrement annoté par un lecteur du XVT siècle, peut-être même un Italien de la cour, qui a couvert le titre et le verso du titre d'inscriptions en italien, en latin, en grec et en français, et accompagne la majorité des pièces d'un bref argument en italien ou leur donne un titre, ajoutant parfois quelques appréciations, et cochant de nombreux vers sententieux par un trait vertical ondulé, les deux derniers ne portent ni marque d'origine ni annotations. Le censeur royal Albert-François Floncel avait ainsi réuni en quelques années une importante bibliothèque italienne, riche de près de 8000 volumes, qui fut dispersée en I774. On y trouvait la quasi totalité des anthologies poétiques italiennes de la Renaissance, dont les Rime diverse (I54), deux tomes réunis en un volume, adjugés 2 livres I2 sols, et les Rime de diversi nobili huomini (I547), adjugés I livre II. ${ }^{\mathrm{I}}$ Un exemplaires des Rime, conservés à Montpellier, porte la signature d'Alfieri et la date d'acquisition 1795, il accompagne la collection pétrarquienne du grand poète piémontais, léguée à la comtesse d'Albany puis au peintre FrançoisXavier Fabre. ${ }^{\text {I6 }}$

On rencontre rarement de tels livres dans les bibliothèques d'érudits, de bibliophiles ou de simples curieux du XVT iècle. Ainsi les bibliothèque de Claude Bellière ou celle du chirurgien bibliophile François Rasse des Neux, 
dans lesquelles se trouvent pourtant plusieurs éditions de Pétrarque et pour la première, un Tebaldeo «in parvo volumine». ${ }^{17}$ On ne mentionne aucune de ces anthologies dans les bibliothèques des secrétaires du roi, et De Thou lui-même, dont la bibliothèque était riche de nombreux poètes italiens, ne possédait que trois recueils d'importance secondaire, les Rime di diversi antichi autori Toscani (Venise 1542), les Rime de gli Academici occulti (Brescia, I568) et la Raccolta d'alcune piacevoli Rime di diversi (Parme, I582), mais aucun recueil vénitien. ${ }^{8}$ Et le cas est assez systématique dans plusieurs autres bibliothèques pour lesquelle l'on dispose d'un inventaire détaillé, et qui comportent les grands classiques de la poésie italienne (Dante, Pétrarque, Bembo) ou française (Ronsard, Desportes). ${ }^{19}$ Les trois recueils conservés dans la riche bibliothèque italienne du cardinal de Granvelle, les Rime diverse (I545), relie en maroquin vert, les Rime di diversi nobili huomini libro II (I547), un exemplaire dans une reliure d'atelier de Giolito, ${ }^{20}$ les Rime di diversi et eccellenti autori (IISG) constituent à cet égard une exception notable; mais ils rappellent que la culture italienne du cardinal, vice-roi de Naples (I57I-I575), formé à Padoue, en relations régulières avec les libraires de Venise par l'intermédiaire de son agent Ruggero de Tassis, était différente de celle de la cour de France. ${ }^{21}$

Ces livres en effet étaient des livres de poètes et d'écrivains, et ils avaient pour eux une fonction utilitaire, accompagnant traités de poétique, dictionnaires et recueils de lieux communs. Ronsard possédait les Rime diverse di molti eccellenti auttori (I546) et le second livre des Rime di diversi nobili huomini (I548) sur lesquels il porta sa signature et qu'il annota vers I5SI, au moment de la composition des Amours. ${ }^{22}$ Le volume, acquis en 1949 par la Bibliothèque Nationale, avait appartenu à Prosper Blanchemain dont la collection avait été mise en vente par le libraire londonien Maggs. Le poète disposa plus tard des Annotationi nel libro della Poetica d'Aristotile d'Alessandro Piccolomini. ${ }^{23}$ Rémy Belleau laissa à sa mort une importante bibliothèque, dans laquelle les recueils poétiques occupaient une place de choix: ${ }^{24}$ les Rime degli Accademici occulti (Brescia, Is68), prisés Io sols, un volume que Vianey estimait être resté inconnu aux poètes français; ${ }^{25}$ les Epigrammi toscani (Mondovi, I570), à s sols, les Sonetti di diversi auttori (Florence, I527), une anthologie d'auteurs anciens estimée s sols, le Libro terzo delle rime di diversi auttori (Venise, I550), à 4 sols, le Libro quarto delle rime (ISII), à deux sols, les Sette libri di Satire (Venise, I570), à 3 sols. Belleau pourtant est considéré comme l'un des poètes de la Pléiade les moins attachés aux modèles italiens. On conserve encore l'exemplaire des Fiori delle Rime que possédait Pierre Tamisier, ainsi que celui des Sette libri di Satire. ${ }^{26}$ Malherbe aurait acquis à Aix l'édition de I579 de l'anthologie de Ruscelli. ${ }^{27}$ L'inventaire de Nicolas Colin, le secrétaire 
à Reims des cardinaux de Lorraine et de Guise, révèle une bibliothèque fort riche, comprenant tous les grands noms de la poésie française et un fonds italien particulièrement important, 70 livres en langue originale et plus de 60 traductions. ${ }^{28}$ Mais Colin était lui-même un traducteur qui laissa, outre ses version d'ouvrages de spiritualité, une traduction du Pastor fido. Trois anthologies poétiques étaient recensées: Rime di diversi. Bologna, Rime delle illustris. Venetia, Rime scelte II. Ven. Elles s'ajoutaient à d'autres recueils poétiques (plusieurs éditions de Pétrarque, les Rime de Dolce, les Sonetti de Sannazar) et prenaient sens à côté de traités sur la langue et d'anthologies épistolaires qui pouvaient lui servir dans ses fonctions. Honoré d'Urfé possédait au moins un semblable recueil. ${ }^{29}$

Dans d'autres bibliothèques ò̀ l'on s'attendrait à les trouver, ces anthologies font défaut; peut-être simplement parce que les inventaires de ces fonds est lacunaire. L'on ne connaît ainsi aucun volume de ce type parmi les livres de Pontus de Tyard, dont on conserve pourtant l'exemplaie annoté des Rime de Pétrarque, témoignant de son approximative maîtrise du texte italien. ${ }^{3 \circ} \mathrm{On}$ n'en connaît pas non plus provenant de la riche bibliothèque de Desportes, alors même que toute la poésie de Desportes révèle une utilisation attentive et régulière de ces anthologies, et que ses livres ont été mieux conservés que ceux des autres écrivains français de son époque. ${ }^{3 \mathrm{I}}$ Des 287 volumes aujourd'bui recensés de sa bibliothèque, imprimés et manuscrits, on connaît 47 livres en italien, dont un seul Pétrarque (Milan, I494), deux éditions de Dante (Amoroso convivio, Venise, Is32, De Vulgari Eloquentia, Paris, I577), Serafino (Venise, I544), Berni (Venise, I545), les Rime de Frangipani (Venise, I566), mais aucune anthologie, alors que les études de sources font apparaître qu'il avait utilisé les principales de celles-ci. Claude Expilly achète, entre I580 et I582, au cours de son séjour italien, de nombreux recueils poétiques, les Rime de Della Casa (I558), de Bembo (I567), de Bernardo Tasso (I560), de Torquato Tasso (I582), indiquant toujours la date de son achat, mais on ne connaît pas les anthologie poétique qu'il aurait possédées. ${ }^{32}$ La lecture de ces anthologies par les poètes de l'époque et leur usage dans la poésie française du temps apparaît ainsi autant par la confrontation des textes et les études des sources, que par de rares données objectives, liées à des exemplaires conservés et annotés, à l'exception remarquable de celui de Ronsard.

\section{Les recueils néo-pétrarquistes et la poésie française}

Le recueil des Rime diverse n'est pas la première anthologie poétique italienne. On connaît les modèles suivis et surtout contestés par les Giolito, qui imposa contre eux un choix élargi, "sovraregionale", au canon florentin. En outre, les Rime diverse répondaient clairement à une initiative éditoriale et à 
des fins commerciales, visant le marché italien, sans réel souci savant ni de correction philologique. Ce fut, sans doute pour ces mêmes raisons, le premier recueil à connaître une véritable réception française. La date de sa parution, I545, est du reste assez emblématique: elle suit la disparition de Marot, elle paraît l'année même où Maurice Scève procure la première édition de Pétrarque donnée à Lyon par Jean de Tournes. Ce n'est pas un hasard alors si le recueil fut utilisé par les poètes de la nouvelle école, Du Bellay en premier lieu, et présenté, même sous forme allusive, comme un des modèles de la réforme poétique qu'ils entendaient imposer et de la nouvelle inventio qu'ils proposaient. Du Bellay notait dans la préface à la première édition de L'Olive:

je confesse avoir imité Petrarque, et non luy seulement, mais aussi l'Arioste et autres modernes Italiens: pource qu'en l'argument que je traicte, ne n'en ay point trouvé de meilleurs. ${ }^{33}$

Revenant sur le sujet dans l'avis au lecteur de la seconde édition, en I550, il précisait cette imitation qui avait suscité quelques réserves, pour la définir plutôt comme la mise en oewvre d'une topique, garantissant sa «naturelle invention» et se situant par rapport aux Italiens dans le même rapport que ceux-ci avec les Latins dont ils étaient les «immitateurs». ${ }^{34}$ Le modèle italien était de surcroit déplacé du plan poétique au plan social: désireux faire de la poésie une activité de gentil-bomme, Du Bellay pouvait se placer sous le patronage aristocratique de Bembo qu'il désignait par allusion:

Certes j'ay grand'honte, quand je voy' le peu d'estime que font les Italiens de nostre poësie en comparaison de la leur: et ne le treuve beaucoup etrange, quand je considere que voluntiers ceux qui ecrivent en la langue toscane sont tous cardinaux mesmes et aultres seigneurs de renom, qui daignent bien prendre la peine d'enrichir leur vulgaire par infinité de beaux escriz. ${ }^{35}$

L'argument ne cessera d'être un des "lieux" de l'apologie du français. La réalité toutefois est plus complexe que le discours polémique dans lequel s'élaborent les nouvelles formes en partie imitée des Italiens. Ronsard lui-même donne dans son exemplaire des Rime diverse un témoignage intéressant. $\dot{A}$ la page 69, il soulignait en effet l'avant dernier vers du sonnet de Giulio Camillo, Tu che secondo l'alta Roma, et notait en marge «Sonet traduit par S. Gelais pour le rois. ${ }^{36}$ L'indication est précieuse, car elle révète le principe d'une émulation, et l'origine du modèle italien, d'origine aulique, qui allait être suivi par Du Bellay: le poète de Mellin de Saint-Gelais encore une fois, en traduisant ce sonnet avant I547 pour celui qui n'était alors que le dauphin, avait joué son rôle, dans le cadre d'une poésie de cour, et suivant une initiative royale. $^{37}$ 
Le premier, Du Bellay utilisa de façon systématique les Rime diverse, probablement d'après la réimpression de I546: l'édition originale de L'Olive compte 20 sonnets sur so qui sont partiellement traduits, adaptés ou composés dans une relation d'imitation partielle avec une pièce du recueil; une autre vingtaine de sonnets tirent leur invention de l'Arioste, sonnets ou Orlando furioso, une dizaine, de Pétrarque; un sonnet semble provenir du tome II des Rime diverse, duquel Du Bellay allait prendre le modèle d'un sonnet fameux des Antiquités de Rome. ${ }^{38}$ Dans l'édition augmentée de L'Olive publiée en I550, 23 sonnets nouveaux provenaient de l'imitation d'une pièce des Rime, également répartis entre les deux premiers tomes; 44 sonnets sur IIS étant ainsi à rattacher au recueil italien. À partir de I550, certains poètes français connaissaient et utilisaient les anthologies italiennes, et les Rime diverse en particulier. Ronsard avait coché dans son exemplaire plusieurs pièces qu'il utilisa pour ses Amours, notant en particulier trois des quatre sonnets de Gesualdo qu'il allait imiter (Qual empio, Né di selvaggio, Verrà mai). Huit sonnets des Amours (I552-I553), sur 221 pièces peuvent être rapportés aux Rime diverse, 3 au tome II, mais I6 au seul Bembo, dont Ronsard devait utiliser un volume séparé, et une majorité de pièces à Pétrarque. ${ }^{39}$ Baïf, puis plus tard Magny firent quelques emprunts à l'anthologie de Giolito. ${ }^{40}$ Desportes encore l'utilisa pour ses Premières CEuvres, en I573, par l'imitation d'un sonnet de Mozarello, ${ }^{4 \mathrm{I}}$ et en I 577 , du sonnet de Gesualdo, Né di selvaggio cuore, déjà imité par Ronsard, ainsi qu'un sonnet d'Amanio, dans le second tome des Rime. ${ }^{42}$

Ces anthologies, qui allaient se multiplier, étaient devenues des outils de travail pour les poètes; ils y trouvaient les ressources formelles et rhétoriques de leur compositions: inventio par le recours à une topique néo-pétrarquiste, dispositio par le recours à une forme, le sonnet. Les néo-pétrarquistes étaient eux-mêmes les utilisateurs de cette inventio et de cette dispositio qui ne leur appartenaient pas mais qu'ils contribuaient à enrichir. Cette fonction purement utilitaire était mise en avant par les éditeurs de ces recueils et elle s'inscrivait dans les modes habituels et légitimes de l'écriture poétique. Dans la préface d'une de ces anthologies, Lodovico Dolce indiquait le profit que pouvaient en retirer les «studiosi della volgar poesia», le plaisir et l'utilité:

non picciola utilità ne potranno essi ritrarre a i componimenti loro da esempio ed imitatione di questi tali. ${ }^{43}$

Les poètes qui savaient les lire disposaient ainsi d'un ensemble de ressources qui leur offraient la possibilité de combinaisons infinies, nouvelles par rapport à la tradition française de Lemaire à Marot, à un moment de renouvellement des formes et de thèmes. Ils acceptaient les ressources de ces anthologies dans 
leur logique même, celle de l'imitation avec variation, et dans leur limite, les anthologies proposaient des suites de pièces isolées. Dans sa formule de la Deffence, évoquant le sonnet comme une «invention italienne», Du Bellay entrevoyait derrière Pétrarque qu'il nommait, une masse confuse de poètes anonymes qui reprenaient indéfiniment un même thème amoureux et ses variations. Selon Chamard, qui renvoyait à Vianey, il s'agirait des poètes de la première moité du siècle, qui s'ingénièrent à reproduire la manière raffinée de Bembo. ${ }^{44}$ Du Bellay, en fait, n'avait aucune conscience des débats italiens ni de leurs subtiles nuances. Il manquait à tous les poètes qu'il imitait une dimension essentielle, la continuité. Seul Pétrarque était connu pour avoir composé un recueil ordonné, constamment édité comme tel, et traduit dans son ensemble dès I548. Dès ces années, et sur ce modèle, les poètes français avaient eux aussi composé des recueils. Les néo-pétrarquistes étaient connus par des pièces isolées, toujours utilisées de façon fragmentaire dans le cadre du recueil en français. Dès leur parution, L'Olive et les Amours, offrirent des médiations et des modèles français assez. forts et convaincants qui, sans remplacer les Italiens, se superposèrent à leur imitation. Ronsard joua pour les jeunes poètes le même rôle que Pétrarque et les néo-pétrarquistes. Les Epithètes de La Porte, publiées en I572, eurent une même importance dans la codification de ce modèle «utile à ceux qui font profession de la Poësie» et ses variations. L'imitation d'une pièce italienne devenait une imitation médiatisée par un premier modèle français et dans une relation subtile d'intertextualité.

Alors qu'il évoquaient Pétrarque dans leurs propres poèmes, qu'ils indiquaient clairement que tel poème était «pris de Pétrarque», les poètes français ne mentionnaient que rarement les néo-pétrarquistes italiens. Leur silence n'était pas le masque du plagiat comme on l'a cru. Du Bellay, dans la préface à L'Olive, reconnaissait au contraire ses emprunts, nommant Pétrarque, l'Arioste et «d'autres modernes Italiens». ${ }^{45}$ Sa prétérition était l'expression même des hiérarchies littéraires, distinguant entre une auctoritas reconnue et valorisante, et les fonctions utilitaires. Dans sa seconde préface, il nommait bien «Cassola Italien», mais pour dénier toute ressemblance entre ses poèmes et les siens, contre ceux qui prétendaient qu'il l'avait imité en concluant ses sonnets de manière épigrammatique, et ainsi minorer la part de l'imitation italienne. Du Bellay prétendait ne pas connaître cet auteur lorsqu'il composait son recueil, avant mars $1549 .{ }^{46}$ De fait, le cavalier Cassola figurait par quatre pièces dans la première édition des Rime diverse, mais sa contributaion avait été supprimée de la seconde édition, dont Du Bellay s'était servi.

Dans son commentaire des Amours, Marc-Antoine Muret voulait à la fois éclairer le recueil de Ronsard dans son détail et le donner à lire dans son ambition savante. ${ }^{47}$ À propos de chaque poème, Muret citait les sources et les références antiques, tirées d'Ovide principalement, qui faisaient du recueil 
moins la confession d'un coeur transi qu'une moderne et originale adaptation d'une longue tradition érudite. À côté des Anciens, Muret plaçait Pétrarque, qu'il nommait ou alléguait plus de quarante fois, parce qu'il était lui aussi une autorité, le garant de cette poésie, dans laquelle il intervenait en personne. En revanche, dans son commentaire, il semblait négliger une autre "source" d'inspiration, les néo-pétrarquistes des Rime diverse ou di diversi, auxquels Ronsard avait fait de nombreux emprunts. Muret mentionnait Bembo à l'occasion de I3 poèmes, l'Arioste six fois: tous les deux étaient reconnus comme des auteurs à par entière. Capilupi et Rainerio, mentionnés à l'occasion d'un ou deux poèmes, ne comptaient guère, et encore moins Gesualdo, à qui Ronsard pourtant avait emprunté la matière de quatre poèmes, et qu'il avait coché dans son exemplaire des Rime. ${ }^{48}$ Muret faisait des allusions assez. précises à ces textes pour que nous puissions être assurés qu'il connaissait l'utilisation qu'en avait faite Ronsard. Son quasi silence n'était pas l'expression de gêne devant ce qu'il ne considérait pas comme un plagiat; la référence à des sonnettistes italiens, probablement indiquée par Ronsard lui-même et non pas laissée à la sagacité du commentateur, ne valait pas, tout simplement la peine d'être autrement précisée.

\section{Modes de lectures et d'utilisation}

L'exemplaire conservé à la Bibliothèque Mazarine, relié en France au milieu du XVT siècle, ainsi que l'attestent les fragments d'un manuscrit plus ancien servant à la forme du dos, porte trace d'une première lecture, antérieure à celle de Du Bouchet: quelques traits verticaux à la plume en marge, épars tout au long des cent premières pages, indiquent des vers isolés; à la page 6I, le lecteur anonyme indique par deux traits les vers 3-5 du sonnet de Giulio Camillo Rugiadose dolcezze. Mais rien n'explique ce choix. Si les Français lurent ces recueils italiens, ils le firent, le plus souvent, pour les utiliser en poètes. L'exemple de Du Bellay et les annotations de Ronsard permettront de proposer trois lignes directrices. Leur lecture en effet était déterminée par une intention pratique et utilitaire.

Une première constatation s'impose. La maîtrise de la langue détermine le rapport au texte italien, et partant les modes de l'imitation. Or nous ignorons tout de la maîtrise de l'italien qu'avait Du Bellay avant son séjour romain. La question jusqu'ici n'a même jamais été posée tant sa réponse semble aller de soi: si Du Bellay avait imité les Italiens, c'est qu'il était capable de les comprendre, et l'imitation des Rime était celle d'un italianisant confirmé. Rien n'est moins sûr. L'exemple de Pontus de Tyard, mis en lumière par V.-L. Saulnier, devrait sur ce point nous inciter à la prudence. Du Bellay, dans sa préface de I550, était très précis sur ce point, si toutefois son argument 
ne servait pas à minorer la part d'imitation de son recueil en dissimulant ses propres capacités linguistiques:

j'ay entendu ce que l'en a peu apprendre la communication familière de mes amis. 49

Affirmant ne pas comprendre l'italien, il devait se faire expliquer les poèmes par certains de ses amis compétents dans cette langue. Quels étaient ces amis? On pensera naturellement à Jacques Peletier, que Du Bellay connaissait depuis I546 pour le moins, et qu'il citait dans la même préface comme celui qui l'avait incité à choisir la forme du sonnet. Peletier, lui même traducteur de douze sonnets de Pétrarque, ${ }^{\circ}$ ne semble pas avoir imité les néo-pétrarquistes recueillis dans les Rime diverse. On pourra aussi penser à Jean Martin, qui venait de traduire l'Arcadia, et surtout à Mellin de Saint-Gelais, tous deux mentionnés avec chaleur dans la préface de I549; la note de Ronsard dans son propre exemplaire confirme ce magistère italianisant du poète de la cour. On rappellera ainsi que les textes poétiques ont été, au XVI siècle, des lieux de l'apprentissage des langues, et les imitations, des exercices linguistiques avant d'être des modes poétiques.

Les analyses très détaillées d'Ernesta Caldarini et de Jo Ann Della Neva concernant L'Olive, ou celles d'Yves Girand à propos des Amours de Magny" permettent d'éclairer, autant que les "sources" proprement dites, les principaux modes d'utilisation des pièces italiennes: il s'agit d'une utilisation diversifiée. Dans ce que les études de sources voyaient traditionnellement comme traduction littérales et simples décalques, nécessairement insignifiants, serviles ou imparfaits, il faut au contraire mettre en évidence les réussites et la qualité de versions qui respectent la disposition et les ornements sans écarts de sens, cherchant à rendre ce que Du Bellay nommait la "grâce" de l'original: ainsi la version que Magny donne d'un sonnet de Castellani, où il parvient à respecter les mots à la rime. La traduction littérale restait un cas limite, probablement une expérience poétique poussée à bout dans toute sa rigueur plutôt qu'une simple étape destinée à fournir matière à variation. ${ }^{52}$ On trouvera ainsi toutes les modes de l'imitation, de la variation et de la sélection, du sonnet intégralement repris au vers isolé. Celui-ci joue comme un détail d'invention et jamais comme un vers "épigraphique", valant référence; cet usage est limité aux vers pris de Pétrarque. Dans L'Olive, on trouve plusieurs de ces emprunts de détail: un vers d'une canzone de Sellaio (sonnet IV'), un vers de Parabosco (sonnet $X$ ), un vers de Coccio (sonnet XLI), un vers de Castellani (sonnet XLVI). Dans tous ces cas, l'invention est plus complexe et procède par contaminatio de deux sources, le vers adapté permettant une variation dans le cadre d'une imitation stricte en forme de traduction d'un sonnet entier, d'Accolti, de l'Arioste, de Tomitano, et de Capilupi. 
Les anthologies italiennes, et au premier chef les Rime diverse confirment pour les Français le prestige et les virtualités d'une forme, le sonnet, même si d'autres formes, le madrigal ou la canzone sont également représentées; ces pièces ne seront pas imitées en tant que telles, et leur matière passera dans des sonnets français. Du Bellay indique nettement ce lien entre les Italiens et le sonnet dans la préface de L'Olive comme dans la Deffense. Cette forme, en France, était alors une forme rare, pratiquée depuis une dizaine d'années à peine et dont il fallait établir les modèles. L'imitation italienne était ainsi l'occasion d'une expérimentation de la structure même du sonnet, de la disposition des rimes, avant leur codification à la française, de certaines dispositions singulières, ainsi le sonnet en anaphore, dont les sonnets LXI, CXLIII et CLXXI des Amours de I553 seront l'exemple remarquable; Pétrarque avait donné le prototype, repris par Gesualdo (sonnet $\mathrm{O}$ viva fiamma), Du Bellay l'avait introduit dans L'Olive (XCVI, pris de Pétrarque).

L'anthologie italienne était vouée à une lecture et à une utilisation fragmentaires, ce qu'attestent à la fois les notes et traits de plume épars dans le volume de Ronsard, sans caractère systématique, et le choix opéré par Du Bellay. Les Rime diverse proposent en effet près d'une centaine de poètes, offrant plus de 600 pièces. Dans la première édition de L'Olive, Du Bellay eut recours à 20 poètes de l'anthologie, répartis dans l'ensemble du volume; il utilisait quatre d'entre eux pour deux sonnets différents, III et XVIII: Guidiccioni (sonnet 3I); XIII et XX: Mozzarello (sonnets I6 et 2); XXXVIII et XL: Coccio (sonnet 4 et 3); XLIV et XLVI: Castellani (sonnet I et canzone I9). L'ordre du recueil italien et celui du recueil français sont bétérogènes; tout au plus pourra-t-on noter des effets de proximité dans les cas de Coccio et de Castellani. À l'anthologie italienne ordonnée selon une logique d'auteurs, s'oppose ainsi le recueil amoureux français, unitaire, suivant une ordonnance thématique et fictionnelle.

Les anthologies italiennes servirent-elles du moins de modèles pour la constitution d'anthologies françaises? Il existait une tradition proprement française de l'anthologie poétique, antérieure aux Rime de I545, et elle relevait d'une même pratique éditoriale autant que poétique. Ce n'est qu'à la fin du siècle que s'opéra une conjonction des deux traditions nationales: le recueil des Fleurs des plus excellents Poetes de ce temps, publié à Paris en I599, renvoie, par son titre aux Fleurs de poesie françoyse de I534, mais aussi aux Fiori delle rime de' Poeti illustri de Ruscelli (I558), et il contient, en plus des pièces de poètes français, les Lagrime di Christo du Tasse données dans leur langue originale.

En lisant plume à la main son exemplaire des Rime diverse, Ronsard, dans un premier temps, alors qu'il était lui-même en train de composer les Amours, notait en marge de certaines pièces, à treize reprises, le nom de Du Bellay, et il identifiait ainsi certains des sonnets que son ami et rival avait 
utilisés pour L'Olive. Il pouvait s'agir d'une curiosité (débusquer les emprunts), un jeu savant auquel se livraient d'autres lecteurs savants, Pasquier comme Henri Estienne. Il powvait aussi, pour le poète, ainsi que le suggère Lebègue, d'éviter de prendre dans le recueil des pièces déjà utilisées par un autre. Ronsard, à l'occasion de plusieurs pièces, indiquait aussi une référence italienne, Bembo à propos d'Annibale Tosco, Pétrarque à propos de trois autres sonnets du même. Enfin, à plusieurs reprises, Ronsard indiquait à propos d'une fable ou d'un nom, son origine antique, Théocrite, Musée, Martial, et il notait un vers de Virgile. Ces mentions, pour fragmentaires qu'elle puissent paraître, indiquent le triple intertexte, antique, italien et français, sur lequel jouait à la fois la "lecture" de l'anthologie de Giolito et la composition d'un recueil de sonnets amoureux par un jeune poète français. Les sonnets LXII de L'Olive, Qui vouldra voir le plus precieux arbre, I et LXIV des Amours, Qui voudra voir comme un Dieu me surmonte et Qui voudra voir dedans une jeunesse se répondent ainsi, via un vers épigrammatique pris de Pétrarque mais aussi via les variations données par Dolce et Parabosco dans les Rime. En revanche, ces allusions, à l'origine de la composition et qui fondaient le rapport d'émulation entre les poètes, ne jouaient pas pour un lecteur moyen du temps, qui ne connaissait pas le recueil de Giolito, sauf lorsqu'elles sont savamment révélées ou dissimulées à travers dénégations et prétéritions par le jeu du paratexte (la préface de L'Olive ou le commentaire de Muret). Plus importante est en revanche la référence à l'intertexte français, et au cours de le seconde moitié du siècle, s'imposera une relation non plus duelle ( $d u$ français à l'italien), mais triangulaire (du français au français via l'italien).

\section{Veronica Gambara entre Desportes et Du Bellay}

En I598, paraissait à Rouen une nowvelle édition de la traduction des Psaumes de David et des Euvres chrestiennes de Desportes. Ce recueil contenait une section de Sonnets spirituels comprenant dix-buit pièces. Dix-sept d'entre elles avaient été publiées dans les Premières CEuvres entre I575 et I587, constituant progressivement une section autonome au sein des oeuvres meslées avant d'être regroupées à la suite des Psaumes. Ces pièces spirituelles étaient à lire dans un contexte lyrique et pénitentiel, contribuant à mettre l'ensemble du recueil profane dans une perspective chrétienne et poétique, béritée de Pétrarque: la matière amoureuse prenait sens au cours d'une mutatio animi liée à la mort du protecteur et à la maladie du poète; elle s'enrichissait d'une meditatio mortis et d'une tension nouvelle entre l'amour profane et l'amour sacré. Desportes concluait son oeuvre par une véritable conversion. L'abbé de Tiron ajouta un dernier sonnet à son recueil, qu'il 
disposa non pas à la suites des autres, mais entre un acte de contrition (sonnet $X I$, publié en I576) et la déploration des vanités terrestres (sonnet XII, publié en I583). Ce nouveau sonnet contribuait à rétablir comme axe de symétrie dans l'ordonnance du recueil le mea culpa du sonnet IX qu'avait provisoirement fait disparaître un sonnet ajouté dans l'addition de 1587. Il introduisait en revanche un élément nouveau, rompant la cohérence de l'énonciation, dans la mesure où tous les autres sonnets étaient des invocations, exhortations ou prières, ou des déplorations. Ce sonnet sous forme d'interrogation oratoire était un acte de foi, affirmant le choix des élus et leur justification par la grâce:

De toute éternité, plein d'amour infinie,

Dieu choisist ses eleus, avant que d'estre nez,

Les fait enfans de grace, et sont predestinez

Pour avoir leur partage en l'eternelle vie.

Apres il les appelle, et benin les convie

A croire que par foy tels biens leur sont donnez:

Les regenere en Christ, les rend illuminez,

Et fait qu'à sa bonté leur ame est tout unie.

Reformez par sa grace ils sont saints et parfaits,

Christ est leur moyenneur, il porte leurs meffaits,

Et d'un nouvel esprit au dedans les inspire.

Or si par tant d'amour il les daigne asseurer,

Quelle amorce du monde, ennuy, mort ou martyre

Peut des bras du Seigneur ses eleuz separer? ${ }^{53}$

La tonalité en apparence très "protestante" de cette pièce, l'emploi de termes ou d'expressions fortement connotés (eleus, predestinez, Christ, Reformez, moyenneur) détonnent dans le contexte mondain des Sonnets spirtuels. Ils pourrait même conduire à mettre en doute l'orthodoxie de l'abbé de Tiron, sauf à admettre que cette pièce, composée par un autre, aurait été publiée à son insu. ${ }^{54}$ Or elle figure dans toutes les éditions à privilège, publiées sous le contrôle de l'auteur, en particulier l'édition revue par lui pour Abel L'Angelier, en I603. Desportes était bien l'auteur du sonnet. La présence de cette pièce singulière dans le recueil s'expliquait en termes poétiques, d'imitation, d'émulation et de réécriture, liés aux sources mêmes de l'invention, plus qu'en termes de dogme.

Dans ses sonnets spirituels, Desportes ne se servait pas moins des ressources offertes par les anthologies poétiques italiennes que dans son oeuvre profane. Joseph Vianey avait identifié les sources de quatorze pièces du recueil. Elles provenaient d'auteurs divers, Amanio, Daniello, Parabosco, Caro, Coppetta, Tansillo, Molza, Bembo, Nigresoli, Sannazar, Dal Pero, et de pièces qui 
n'avaient pas toujours un sujet religieux, tirées des Rime diverse de I545, des Rime di diversi de 1547, des Fiori delle rime de 1558 , des Rime scelte de I565, recueils que Desportes avait utilisés dès I577. On ajoutera à ces sources celle du sonnet III, en l'occurrence un quatrain imité de Vittoria Colonna, ${ }^{55}$ et surtout celle du sonnet XII, entièrement adapté d'un sonnet de Veronica Gambara.

Veronica Gambara, en compagnie de Vittoria Colonna et de Gaspara Stampa, figure en bonne place dans le catalogue des dames illustres de la Renaissance italienne. ${ }^{56}$ Elle laissa une oeuvre poétique rare mais de haute qualité, composée sur plusieurs décennies, éparse et publiée de façon fragmentaire dans différentes anthologies vénitiennes, et qui ne fut réunie qu'en 1769 par les soins de Felice Rizzardi. Il s'agit d'un ensemble de 54 compositions amoureuses, sonnets, canzoni et madrigaux, auxquelles s'ajoutent des sonnets in risposta ou d'éloge, ainsi que quatre sonnets spirituels composés entre I538 et I540. Les deux premiers évoquent les mystères de la divine maternité de Marie et de l'incarnation, le dernier est une prière à Dieu «affinchè la aiuti dalla guerra che le muovono $i$ sensì. Le troisième sonnet, d'un schéma métrique particulier (les rimes des tercets sont en $C D E D E C$ ), utilisé une seule autre fois par l'auteur, est consacré à la justification par la grâce,

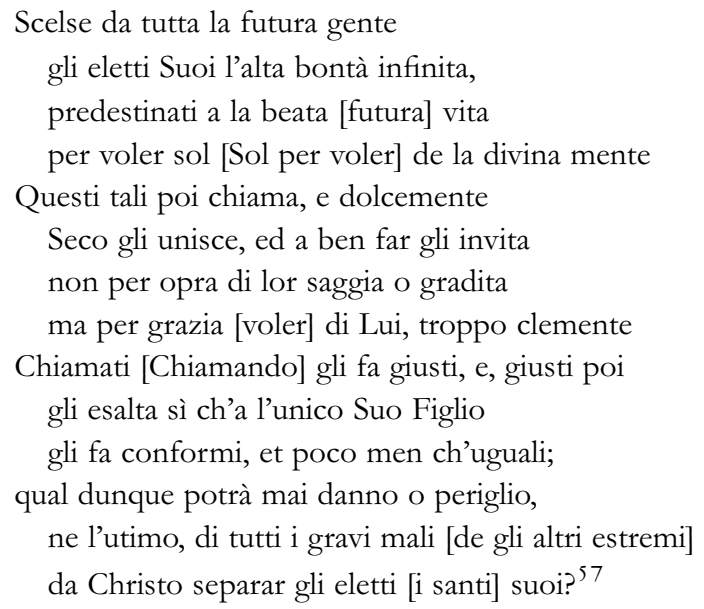

Le sonnet est une paraphrase de la lettre de saint Paul, Romains, 8, 29-30, dont il reprend les termes avec précision: "quos praescivit, et praedestinavit conformes fieri imaginis Filii sui; quos autem praedestinavit, hos et vocavit: et quos vocavit, hos et justificavit: quos autem justificavit, illos et glorificavit». Le ternier tercet paraphrase 8, 35: «quis ergo nos separabit a charitate Christi?». 
Cette pièce est d'une orthodoxie catholique reconnue, et sera encore considérée comme telle un siècle et demi plus tard, dans le milieu fort dévôt de la cour de Naples. ${ }^{58}$

On connaît de ce sonnet, qui date probablement des dernières années de Veronica Gambara, deux versions manuscrites contemporaines, mais alographes. ${ }^{59}$ Il est surtout, de toutes les pièces de Veronica Gambara, l'une des plus diffusées au XVI siècle, à travers plusieurs éditions. Il paraît en effet pour la première fois en 1547 dans le second volume des Rime di diversi et dans la seconde édition de I548, ${ }^{60}$ seule pièce de l'auteur; réimprimé en I550 dans le Libro primo delle rime spirituali, avec un autre sonnet, Vinca gli sdegni e l'odio antico, attribué à la poétesse mais en fait de Vittoria Colonna. ${ }^{61}$ Ruscelli le reprend, avec seize autre pièces de l'auteur, dans ses Rime di diversi eccellenti autori bresciani, puis en Is 8 dans ses Fiori delle rime de' poeti illustri. ${ }^{62}$ Domenichi le choisit l'année suivante avec vingt autres pièces dans ses Rime diverse d'alcune nobilissime et virtuose donne. ${ }^{3}$ Il paraît une dernière fois au XVT siècle, en I574, à Naples, dans les Rime spirituali di diversi eccellenti poeti toscani de Giovan Battista Vitale. ${ }^{64}$ Ces éditions proposent un texte moins bon du point de vue poétique, introduisant plusieurs répétitions de termes, et moins précis du point de vue religieux que le manuscrit, qui introduit la notion de gratia (v. 8), transformée en voler dans le texte imprimé, et conclut sur les santi (v. I4), là où les éditions donneront eletti, répétant le terme employé au v. $I$.

Avant d'être imité par Desportes, le sonnet de Veronica Gambara fut connu en France par le texte imprimé dans l'anthologie de I547. Il fut imité une première fois en français par Du Bellay dans L'Olive. ${ }^{65} D u$ Bellay évoquait le choix des âmes élues, leur ascension au sein des beautés éternelles, leur justification par la grâce et leur salut. La paraphrase paulinienne de la poétesse italienne suscita ensuite l'émulation de Simon Goulart. Celui-ci suivit avec précision son modèle dans un sonnet de la Suite des imitations chrestiennes, un recueil de 198 sonnets publiés en 1574 dans l'édition des Poèmes chrestiens de B. de Montmeja et autres divers auteurs, procurée par Ph[ilippe] de Pas. ${ }^{66}$ Le calviniste Goulart ne traduit pas predestinati. L'épitre de saint Paul dont Veronica Gambara avait tiré son argument fit la matière des sonnets XXIII-XXIX de la Muse chrestienne de Pierre Poupo. ${ }^{67}$ L'italianisme apparent de Desportes reposait ainsi sur un plus subtil intertexte français. Le recueil des sonnets spirituels de Desportes en effet est placé sous le patronage de Du Bellay. Le deuxième sonnet, en fait le premier publié, dès I575, est en effet une claire variation sur le sonnet CXIII de L'Olive, Si nostre vie est moins qu'une journée, que Desportes avait enrichie en revenant à la source italienne, le sonnet de Daniello, Se 'l viver nostro è 
breve oscuro giorno, lu dans les Rime diverse de I545. Le troisième sonnet du recueil, dont on ne connaissait pas la source italienne, reprend partiellement le sonnet CVIII de L'Olive, le quatrième, le sonnet CIX, lui-même imité de Pétrarque. Desportes trouvait à la fin de L'Olive un groupe de sept sonnets dans lesquels Du Bellay corrigeait le lyrisme amoureux de son recueil en lyrisme spirituel. Il prenait à son prédécesseur un modèle de poésie chrétienne, ses arguments, ses "lieux" et ses formes rhétoriques, en les enrichissant de la haute poésie des Psaumes et du Livre de Job.

Le sonnet de Du Bellay avait été repris dans L'CEuvre chrestienne, une anthologie publiée à Lyon en I58I. ${ }^{68}$ Ce recueil, dù à un certain I.D., accordait à Desportes une place notable, en proposant les I2 sonnets chrestiens publiés à cette date. Ces mêmes pièces de Desportes et de Du Bellay étaient à nouveau publiés dans La Muse chrestienne. ${ }^{69}$ Ce recueil, édité par un autre anonyme, I.C.T., sous le contrôle de Desportes et de Baïf "ausquels ce recueil de poesies a esté communiqué et mis en lumière de leur consentement» était adressé au duc de Joyeuse, le protecteur de Desportes et publié dans un clair dessein anti-protestant. Les pièces étaient classées par genre, et les sonnets eux-mêmes étaient répartis par arguments; les sonnets spirituels de Desportes, disposés en alternance avec des sonnets de Du Bellay de même genre et de même argument, étaient distingués d'un point de vue rhétorique entre invocations et prières, et entièrement redistribués selon une disposition mise en évidece par les titres: les sept premiers sonnets étant présentés comme des «invocations de la miséricorde et grace de Dieu pour estre delivré des concupiscences de la chair et exciter son ame aux choses spirituelles», les quatre sonnets suivants, "prieres faictes à Dieu pour obtenir pardon des fautes commises contre sa majesté, appaiser son ire, et implorer son secours et misericorde». Le sonnet de Du Bellay était rangé sous l'argument «De l'espérance qu'il faut avoir en Dieu». ${ }^{70}$ C'est dans ce contexte qu'il convient de lire le sonnet XII de Desportes; loin d'être une tardive invention ou une interpolation venant compliquer l'ordonnance du recueil, il trouvait sa raison dans la relation à Du Bellay, et son prototype dans le sonnet CXIII de L'Olive, Dedans le clos des occultes idées, corrigé et précisé par un retour au sonnet original de Veronica Gambara, dont Desportes rendait la lettre paulinienne. Desportes avait publié les sonnets imités de L'Olive en I575 (CXIII), en I576 (sonnet CIX), en I577 (sonnet CVIII). La réécriture du sonnet CXII à la lumière de l'original italien ne fut publiée que vingt-trois ans plus tard. Avait-elle été composée à cette date seulement? En fait, Desportes, soucieux de mettre à jour ses recueils afin de justifier le privilège qui les protégeait, revenait à ses modèles français et italiens, et il ajoutait un sonnet composé bien plus tôt, vers I575, en même temps que les précédents, et dans un même contexte. 
Les Français semblent ne pas avoir été sensibles aux distinctions des recueils, dans lesquels les poètes sont classés par école et par origine géographique. Ils ne se mettaient pas "à l'école" de Tebaldeo ou d'Angelo di Costanzo, contrairement à ce qu'affirmait Vianey, dans une interprétation purement chronologique de l'imitation. Leur poésie suivait les contraintes et les modes du goût national, même s'ils continuaient à découvrir dans le secret de leur cabinet de nouveaux auteurs, objets de leur imitation, Tansillo, le Tasse ou Guarini.

Jean Balsamo 


\section{Jean Balsamo}

I. Rime diverse di molti eccellentissimi autori [Venise, Giolito, I 545], a cura di F. Tomasi e P. Zaja, Torino, Editioni RES, 200 I une bonne introduction au recueil est donnée par M. Bianco et E. Strada, "I più vaghi e i più soavi fiori». Studi sulle antologie di lirica del Cinquecento, Edizioni dell'Orso, Alessandria, 2000.

2. J. Vianey, Le Pétrarquisme en France au $X V T^{e}$ siècle, Paris-Montpellier, I 909. Vianey avait été précédé dans ses recherches par M. Pieri, Le Pétrarquisme au XVT siècle ou l'influence de Pétrarque sur la Pléiade française, Marseille, I 896.

3. On se reportera, pour l'étude de cette tradition à notre étude Les Rencontres des Muses. Italianisme et anti-italianisme en France à la fin du XVT siècle, Genève, Slatkine, I 992.

4. Un tableau de cette "influence" est donné par J. P. Barbier, Ma Bibliothèque poétique, t. III, Genève, Droz, I994, pp. I7-20.

5. Joachim Du Bellay, La Deffence et illustration de la Langue Françoyse [i 549], II, 4, éd. H. Chamard, Paris, STFM, rééd. I997, p. I 22.

6. Voir Y. Giraud, Traducteur servile ou adaptateur intelligent: Olivier de Magny et ses modèles italiens, dans Les Fruits de la saison. Mélanges André Gendre, Université de Neuchâtel-Editions Droz, Genève, 2000, p. 247-57.

7. Voir J. Lavaud, Un poète de cour au temps des derniers Valois: Philippe Desportes, Paris, Droz, I936, p. 175-89 et pp. 275-85; pour un examen critique de cette question, notre Philippe Desportes (I546-1606). Un poète presque parfait entre Renaissance et classicisme, Paris, Klincksieck, 2000, pp. 7-I6.

8. Voir Fr. Flamini, I plagi di Desportes, dans Studi di storia letteraria e straniera, Livorno, I 895 , pp. 346-68 et 433-39; H. Vaganay, Un modèle de Desportes non signalé encore: Panfilo Sasso, «Revue d'Histoire littéraire de la France», X (1903), pp. 277-82; J. Vianey, Une rencontre des Muses de France et d'Italie demeurée inédite, "Revue d'Histoire littéraire de la France», XIII (I906), pp. 92-Ioo; L. E. Kastner, Desportes et Angelo di Costanzo, «Revue d'Histoire littéraire de la France», XV ( I 908 ), pp. I I 3-I 8; J. Vianey, Desportes et Angelo di Costanzo, «Revue d'Histoire littéraire de la France», XV (I908), pp. 330-3 I; L.-E. Kastner, Desportes et Guarini, «Revue d'Histoire littéraire de la France», XVII (I9I0), pp. I 24-3 I.

9. A. Pizzorusso, Il petrarchismo di Desportes, «Studi petrarcheschi», V (1952), pp. 237-97; V. E. Graham, Some undiscovered Sources of Desportes, «French Studies», X (1956), p. I 23 et suivantes.

ı. Voir Babier, Ma Bibliothèque cit., p. 20, ainsi que nos Rencontres des Muses cit., pp. 234-46.

i i. Philippe Desportes, Les Amours de Diane, éd. H. Vaganay, Lyon, I928, p. xx.

I 2. H. Chamard, Joachim du Bellay, Lille, I900, p. I76.

I 3. A. H. Schutz, Vernacular Books in Parisian private Libraries of the Sixteenth Century according to the notarial Inventories, Chapell Hill, Univ. of Noth Carolina Press, I 95 5, n ${ }^{\circ} 6$.

I4. Sur les relations entre libraires parisiens et libraires vénitiens, voir A. Parent, Les Métiers du livre à Paris au XVT siècle (I535-I560), Genève, Droz, I 974, pp. I 5 5-56.

I 5. Catalogo della libreria Floncel, Paris, Cressonnier, I774, p. 289 et suivantes, lots 3974 à 4043. 


\section{PoÈtes FRANÇAIS ET ANTHOLOGIES ITALIENNES}

I6. Sur les livres d'Alfieri conservés à Montpellier, voir notre étude Quelques remarques sur les collections d'éditions anciennes de Pétrarque conservées en France, dans Dynamique d'une expansion culturelle. Pétrarque en Europe $X I V^{e}-X X^{e}$ siècle, Actes du colloque (Turin-Chambéry, I995), Paris, Champion, 2001, en particulier pp. 96-97.

17. L. Auvray, La bibliothèque de Claude Bellièvre, dans Mélanges E. Picot, Paris, i9i 3, t. II, pp. 333-64; J. Veyrin-Forrer, Provenances italiennes dans la bibliothèque de François Rasse des Neux, dans Libri, tipografi, biblioteche. Ricerche storiche dedicate a Luigi Balsamo, Firenze, Olschki, I997, pp. $385-98$.

I 8. Voir H. Michau, Les bibliothèques des secrétaires du Roi an XVT siècle, «Bibliothèque de l'École des Chartes», CXXVI (I968), pp. 333-76. Catalogus bibliothecae Thuanae, éd. Jos. Quesnel, Paris, I679, p. 303.

I 9. Par exemple celle des Du Prat, voir M. Connat - J. Megret, Inventaire de la bibliothèque des Du Prat, «Bibliothèque d'Humanisme et Renaissance», III (I943), pp. 72-I 28, ou d'Antoine de Saint-Sulpice, évêque de Cahors, voir N. Marzac, The Library of a French Bishop in the late XVIth Century, Paris, CNRS, I974.

20. Voir J.-M. Chatelain, Les reliures italiennes de la bibliothèque d'Antoine Perrenot de Granvelle, dans Les Granvelle et l'Italie au XVT siècle. Le mécénat d'une famille, Besançon, Cètre, I996, pp. 78-94 et ill. 2 .

2 I. Voir M. Discours, La bibliothèque d'un cardinal de la Renaissance, dans Les Granvelle et l'Italie cit., pp. 43-70.

22. Le volume est conservé à la BnF. Voir P. Laumonier, Sur la bibliothèque de Ronsard, «Revue du Seizième siècle», XIV (I927), pp. 314-35; R. Lebègue, Un volume de vers italiens annoté par Ronsard, «Bulletin du Bibliophile», I95 I, pp. 273-80.

23. Voir J. Labaste, Un nouveau livre de la bibliothèque de Ronsard, «Bibliothèque d'Humanisme et Renaissance», XLVII (1985), pp. 6i 3-i 8.

24. Voir M. Connat, Mort et testament de Remi Belleau, «Bibliothèque d'Humanisme et Renaissance», VI (1945), pp. 328-56.

25. Vianey, Le Pétrarquisme cit., p. 385.

26. Exemplaire conservé à la Bibliothèque municipale de Troyes.

27. R. Lebègue, La bibliothèque de Malberbe, «Revue française d'histoire du livre», p. 585.

28. Voir H. Jadart, Inventaire du mobilier et de la bibliothèque de Nicolas Colin, extrait de la «Revue de Champagne et de Brie», Arcis-sur-Aube, I 892.

29. Un exemplaire d'une anthologie incomplète du titre et reliée avec la Faustina de Muzio (Venise, I 560), portant l'ex-libris d'Honoré d'Urfé et la date de I6 I I figurait au catalogue de la librairie Les Argonautes, octobre I 98 I, n ${ }^{\circ}$ I 2 I.

30. Voir V.-L. Saulnier, Maurice Scève et Pontus de Tyard: deux notes sur le pétrarquisme de Pontus, «Revue de littérature comparée», XXII (I948), pp. 267-72.

3 I. Voir I. de Conihout, Du nouveau sur la bibliothèque de Desportes, dans Philippe Desportes 


\section{Jean Balsamo}

(I546-1606), éd. J. Balsamo, Paris, Klincksieck, 2000, pp. I 2 I-60. Il faut ajouter à la liste l'Historia di Parma de Bonaventura Angeli, du catalogue de la librairie Sourget, XXVIII, $\mathrm{n}^{\circ} 6 \mathrm{I}$.

32. Voir A. Preda, Montaigne et Claude Expilly, «Montaigne Studies», XIII (2001), pp. I87206.

33. Joachim Du Bellay, L’Olive [i 549], éd. H. Chamard, Paris, STFM, 1908, rééd. i996, p. 8 .

34. Du Bellay, L'Olive, p. 2 I.

35. Du Bellay, L'Olive, p. i6.

36. Lebègue, Un volume de vers italiens cit., p. 275 ; Rime diverse, p. 65.

37. Mellin de Saint-Gelais, Sonnets, éd. L. Zilli, Genève, Droz, i990, pp. 2 i-22. Le sonnet est connu par différents manuscrits.

38. Le sonnet Sacrez costaux, imité du sonnet Superbi colli, attribué à Castiglione, Rime di diversi, t. II, I 548, f. I 32; voir A. Morel-Fatio, Histoire d'un sonnet, dans Revue d'Histoire Littéraire de la France, I 894, pp. 97-102.

39. Nous renvoyons à l'annotation de l'édition des Amours procurée par A. Gendre, Paris, Librairie générale française, I993.

40. Jean-Antoine de Baïf, Les Amours, Paris, Veuve La Porte, I 55 2, p. 3; chanson Desja Phebus, prise de Salvi, Già per tornar, Rime diverse, I 548, p. 305.

4I. Vianey, Le Pétrarquisme cit., p. 235.

42. Philippe Desportes, Les Premières oeuvres, Paris, M. Patisson, i 577, sonnet Ny les desdains de son jeune courage, f. 4 et sonnet Je sçay qu'ell'ont des yeux, f. 43 .

43. Rime di diversi eccellenti autori raccolte dai libri da noi altre volte impressi, Venise, Giolito de' Ferrari, I 553 , épître de Lodovico Dolce, f. * 3.

44. Joachim Du Bellay, La Deffence, p. I 22 note. Dans son édition de la même oeuvre, L. Terreaux mentionne «les poètes connus en particulier dans les anthologies vénitiennes de Giolitto», Paris, Bordas, I972, p. 76 note.

45. Du Bellay, L'Olive, p. 8; nous soulignons.

46. Du Bellay, L'Olive, p. 20.

47. Les Amours de P. de Ronsard nouvellement augmentez.par lui et commentez.par Marc Antoine de Muret, Paris, Veuve La Porte, I 553 ; éd. moderne par C. de Buzon - P. Martin, Paris, Didier Érudition, I999.

48. Les Amours, sonnet XLI (Capilupi), sonnets xLIII et CXLvi (Rainerio); voir également E. Caldarini, A propos du sonnet CVIII de Ronsard, «Bibliothèque d'Humanisme et Renaissance», XXVII (1965), pp. 653-54. Les sonnets LVI, LVII, CLXXI et CLXXIII viennent de sonnets de Gesualdo, cochés par Ronsard dans son exemplaire des Rime, pp. 27-29. 


\section{PoÈTES FRANÇAIS ET ANTHOLOGIES ITALIENNES}

49. Du Bellay, L'Olive, p. I 2.

50. Voir sur ce point M. Françon, Pétrarquisme et néoplatonisme chez Peletier, «Italica», XXXVI (1959), pp. 28-35, ainsi que les remarque de J. Ch. Monferran dans l'introduction à son édition de L'Amour des amours, Paris, STFM, i996, p. Xxiv-Xxxvir.

5 I. Sur ces auteurs, voir E. Caldarini, Nuove fonti italiane dell'Olive, «Bibliothèque d'Humanisme et Renaissance», XXVII (1965), pp. 396-434; J. A. Della Neva, Variations in Minor Key. Du Bellay's Imitations of Giolito Anthology Poets, «French Forum», XIV (1989), pp. 133-46.

52. Rime, p. 46 Omai sott'altro cielo; Giraud, Traducteur servile ou adaptateur intelligent cit., p. 249.

53. Philippe Desportes, Sonnets spirituels, xiI, dans Poesies chrestiennes (I 598), Paris, A. L'Angelier, 1603 , p. 46.

54. Voir Y. Giraud, Les Oeuvres spirituelles des Desportes, dans Philippe Desportes cit., pp. 235-50.

5 5. Voir J. W. Dickinson, Vittoria Colonna, Philippe Desportes and William Alabaster, «Revue de Littérature comparée», XXXV (I961), pp. i I 2-I4.

56. Voir Veronica Gambara e la poesia del suo tempo nell'Italia settentrionale, éd. C. Bozzetti, P. Gibellini, E. Sandal, Firenze, Olschki, I 989.

57. Veronica Gambara, Le Rime, sonnet 57, éd. A. Bullock, Firenze, Olschki, i 995, p. i 57. Nous indiquons les variantes entre crochets.

58. Le sonnet est publié dans les Rime delle Signore Lucrezia Marinella, Veronica Gambara ed Isabella della Morra, Naples, Bulifon, I693, II, p. I 2. Il existe une édition complète des oeuvres de Véronica Gambara, procurée par Felice Rizzardi, en 1759.

59. Florence, BNC, cod. Mgl. VIII. I I 78, f. I 85 ; Foligno, cod. 280 (B.V.8).

6o. Rime di diversi nobili buomini et eccellenti poeti nella lingua thoscana, libro secondo, Venise, G. Giolito de’ Ferrari, I 547 , f. I I 3 v; éd. I 548 , f. i I 2 v.

6. Libro primo delle rime spirtuali, Venise, al segno della Speranza, i 550 , f. 2 I v.

62. Rime di diversi eccellenti autori bresciani, éd. G. B. Ruscelli, Venise, P. Pietrasanta, I 553, p. I6, seconde émission en I 554 .

63. Rime diverse d'alcune nobilissime, et virtuosissime donne, éd. Lodovico Dominichi, Lucca, V. Busdragho, I 559 , p. I 58.

64. Rime spirituali di diversi eccellenti poeti toscani, éd. Giovan Battista Vitale, Napoli, H. Salviani, I 574 , f. $67 \mathrm{~V}$.

65. Joachim Du Bellay, L'Olive, p. i 2 I et commentaire p. 200-20 I. La source du sonnet de Du Bellay a été identifiée par R. V. Merrill, Du Bellay's Olive CXII and the Rime diverse, «Modern Language Notes», LX (1945), pp. 527-30.

66. Suite des Imitations chrestiennes, dans Poemes chrestiens de B. de Montmeja et autres divers auteurs, éd. Ph. de Pas, s.l., I 574 , f. 198. 


\section{Jean Balsamo}

67. Pierre Poupo, La Muse chrestienne, éd. A. Mantéro, Paris, STFM, i 997, pp. 32-40.

68. L'Euvre chrestienne de tous les Pö̈tes François, Lyon, L. Cloquemin pour Paulin Bianchi, s.d. [I 58I]; voir Fr. Lachèvre, Bibliographie des recueils collectifs de poésies du XVT siècle, Paris, Champion, I922, p. 2 Io.

69. La Muse Chrestienne ou recueil de poësies Chrestiennes tirées des principaux Poëtes François, Paris, G. Mallot, I 58 2; voir Lachèvre, Bibliographie cit., pp. 2 I I-I 3, et Barbier, Ma Bibliothèque cit., t. II, pp. 306-09.

70. La Muse Chrestienne, f. $288 \mathrm{v}$. 